TRANSACTIONS OF THE

AMERICAN MATHEMATICAL SOCIETY

Volume 188 , Is sue 2,1974

\title{
A MATRIX REPRESENTATION FOR ASSOCIATIVE ALGEBRAS. I
}

\author{
BY \\ JACQUES LEWIN( ${ }^{1}$ )
}

ABSTRACT. Let $F$ be a mixed free algebra on a set $X$ over the field $K$. Let $U, V$ be two ideals of $F$, and $\{\delta(x),(x \in X)\}$ a basis for a free $(F / U, F / V$ bimodule $T$. Then the map $x-\left(\begin{array}{cc}x+V & 0 \\ \delta(x) & x+U\end{array}\right)$ induces an injective homomorphism $F / U V \rightarrow\left({ }_{T}^{F} / V \quad F / U\right)$. If $F / U$ and $F / V$ are embeddable in matrices over a commutative algebra, so is $F / U V$. Some special cases are investigated and it is shown that a PI algebra with nilpotent radical satisfies all identities of some full matrix al gebra.

I. Introduction. In 1939, W. Magnus [16] proved that if $G$ is a free group with a free set $X$ of generators and $R$ is a normal subgroup of $G$, then the group $G /[R, R]$ has a natural faithful representation in two-by-two matrices. The embedding, now called the Magnus representation, maps the coset $x[R, R]$ to the matrix

$$
\left(\begin{array}{ll}
x R & 0 \\
t_{x} & 1
\end{array}\right),
$$

where $t_{x}$ is a free generator for a free $F / R$ module. This theorem has found wide application. It is, for example, a basic tool in the study of free metabelian groups.

In this paper we give an analogous representation for $F$, where $F$ is the free product of a free algebra on a set $Z$ and of the group algebra of a free group $G$ on a set $Y$ over the field $K:$ if $U, V$ are two ideals of $F$, we embed $F / U V$ into the $K$-algebra $\left({ }_{T} / V{ }_{F} / U\right)$, where $T$ is now a free $(F / V, F / U)$-bimodule with basis $\{\delta(x) ; x \in Z \cup Y\}$. The embedding is given by $x+U V \rightarrow\left(\begin{array}{cc}x+V & 0 \\ \delta(x) & x+U\end{array}\right)$.

Magnus' theorem can be stated in the language of Fox's free differential calculus [7]: an element $w \in G$ is in $[R, R]$ if and only if its Fox derivative, taken modulo $R$, is zero. A similar situation obtains here. The map $x \rightarrow \delta(x)$ defines a derivation $\delta: F \rightarrow T$. If $w \in U \cap V$, then $w \in U V$ if and only if $\delta(w)=0$. The proofs of various versions of the representation theorem occupy \$III.

Received by the editors March 2, 1972. $20 \mathrm{H} 20$.

AMS (MOS) subject classifications (1970). Primary 16A06, 16A38, 16442; Secondary

Key words and phrases. Free algebra, universal derivation, embedding in matrices, matrix identities, Noetherian PI algebras, Abelian-by-nilpotent groups.

(1) This work was supported by NSF grants GP-8094 and GP-33050. 
It is worthy of note that if $F$ is the free group algebra $K G, x$ the augmentation ideal of the normal subgroup $R, g$ the augmentation ideal of $G$, then $\delta$ is essentially the Fox derivative taken modulo $R$ and hence our representation reduces to the Magnus representation. In $\$ I V$ we use this to represent some other groups.

Call a $K$-algebra $R$ embeddable in matrices if there is a $K$-monomorphism of $R$ into $n \times n$ matrices over a commutative algebra. We show in \$IV that if $F / U$ and $F / V$ are both embeddable in matrices, so is $F / U V$. In particular if $t_{n}$ is the ideal of identities of $n \times n$ matrices over an infinite field $K$, then $F / t_{n}^{r}$ is embeddable in matrices. It follows that a PI algebra $R$ with nilpotent upper nil radical satisfies all the identities of some full matrix algebra over $K$. In a more special case we show that if the square of the commutator ideal of $R$ is zero, then $R$ is embeddable in matrices. This does not generalize, as an example of Small [17] shows.

The last section is devoted to proving two results on varieties (in the sense of universal algebra) of PI algebras over an infinite field. We show how the proof of a theorem of D. Cohen [4] can be adapted to prove that a free algebra has ACC on $T$-ideals which contain $t_{1}^{2}$. We also show that if, in a variety, finitely generated algebras are right (left) Noetherian, then finitely generated algebras are finitely presented.

The exposition of this paper owes much to G. Bergman. He read a first version and is responsible for numerous improvements. In particular, the present proof of the main theorem is due to him. The universal approach presented here cuts through much of the combinatorial details.

II. Preliminaries. Let $S$ be a free semigroup on a set $Z$ of free generators and let $G$ be a free group on a set $Y$ of free generators. Let $X=Z \cup Y$, let $M$ be the free product as monoids of $S$ and $G$ and let $F$ be the monoid algebra of $M$ over the field $K$. We write $F$ as $F=K\langle X\rangle$, omitting from the notation which elements of $X$ have inverses, and we call $F$ a mixed free algebra on the set $X$.

Let $R^{\prime}, R^{\prime \prime}$ be $K$-algebras and $T$ an $\left(R^{\prime}, R^{\prime \prime}\right)$-bimodule. (All bimodules over $K$-algebras are understood to have the same $K$ vector space structure on the right and left, i.e. to be $R^{\prime \text { opp }} \otimes_{K} R^{\prime \prime}$ modules. Whenever necessary, we will consider them as such.) The set of matrices $\left\{\left(r_{t}^{\prime \prime}, r^{\prime}\right)\right\}$ with $r^{\prime \prime} \in R^{\prime \prime}, r^{\prime} \in R^{\prime}, t \in T$ is easily seen to be a $K$-algebra under the usual matrix operations. We denote this algebra by $\left({ }_{T}^{R^{\prime \prime}}{ }_{R^{\prime}}^{0}\right)$. Note that if $r^{\prime}, r^{\prime \prime}$ are invertible in $R^{\prime}, R^{\prime \prime}$ respectively then, for any $t \in T$, the matrix $\left(r_{t}^{r^{\prime \prime}}, r^{\prime}\right)$ is invertible with inverse

$$
\left(\begin{array}{ll}
r^{\prime \prime-1} & 0 \\
-r^{\prime-1} t r^{\prime \prime} & r^{\prime-1}
\end{array}\right) .
$$


Throughout the rest of the paper we adopt the following convention. If $R, R^{\prime}, R^{\prime \prime}$ are $K$-algebras (over the field $K$ ) we shall understand that there are given $K$-algebra homomorphisms $r \rightarrow r^{\prime}, r \rightarrow r^{\prime \prime}$ of $R$ into $R^{\prime}$ and $R^{\prime \prime}$. Let then $R, R^{\prime}, R^{\prime \prime}$ be $K$-algebras and $T$ an $\left(R^{\prime}, R^{\prime \prime}\right)$-bimodule. Let $\delta: R \rightarrow T$ be any setmap. The following is well known (see e.g. [10, p. 177]):

Lemma 1. The following conditions are equivalent:

(i) the map

$$
r \rightarrow\left(\begin{array}{cc}
r^{\prime \prime} & 0 \\
\delta(r) & r^{\prime}
\end{array}\right) \text { is a K-algebra bomomorpbism } R \rightarrow\left(\begin{array}{ll}
R^{\prime \prime} & 0 \\
T & R^{\prime}
\end{array}\right) \text {. }
$$

(ii) $\delta$ is a K-derivation; i.e. $\delta$ is K-linear and for $a l l a, b$ in $R, \delta(a b)=$ $a^{\prime} \delta(b)+\delta(a) b^{\prime \prime}$.

From Lemma 1 and the remark on invertibility of matrices, we obtain immediately

Lemma 2. Let $R=K\langle X\rangle$ be the mixed free algebra on $X$, and $T$ an $\left(R^{\prime}, R^{\prime \prime}\right)$ bimodule. Then any map of $X$ into $T$ extends to a derivation of $K\langle X\rangle$ into $T$. $\square$

III. Universal derivations and the representation.

1. The proofs presented here are Bergman's. The main result of this section will be obtained by a noncomputational homological argument in a forthcoming paper by Bergman and Dicks [3].

Let $R, R^{\prime}, R^{\prime \prime}$ be $K$-algebras and $U, V$ the kernels of the given maps $R \rightarrow R^{\prime}$, $R \rightarrow R^{\prime \prime}$. Our problem is to determine the possible kernels $N$ of the maps given in part (i) of Lemma 1. Since $N=U \cap V \cap \operatorname{Ker} \delta$, and

$$
\left(\begin{array}{ll}
R^{\prime \prime} & 0 \\
T & 0
\end{array}\right)\left(\begin{array}{ll}
0 & 0 \\
T & R^{\prime}
\end{array}\right)=0
$$

it is easy to see that $U \cap V \supseteq N \supseteq U V$. We are most interested in finding $T$ and $\delta$ such that the lower bound $N=U V$ is realized.

By universal algebra, there exists a universal $\left(R^{\prime}, R^{n}\right)$-bimodule $T=$ $T\left(R ; R^{\prime}, R^{\prime \prime}\right)$, with a universal derivation $\delta: R \rightarrow T$. More specifically, we have

Lemma 3. Let $R, R^{\prime}, R^{\prime \prime}, T\left(R ; R^{\prime}, R^{\prime \prime}\right)$ be as above.

(i) $T\left(R ; R^{\prime}, R^{\prime \prime}\right)$ is the $\left(R^{\prime}, R^{\prime \prime}\right)$-bimodule $T$ defined by generators $\delta(x)$ $(x \in R)$ and witb relations $\delta(a x+\beta y)=\alpha \delta(x)+\beta \delta(y)$ and $\delta(x y)=\delta(x) y^{\prime \prime}+$ $x^{\prime} \delta(y)(x, y \in R, a, \beta \in K)$.

(ii) If $X$ and $H$ are generators and relations for $R$ as a K-algebra, then $T\left(R ; R^{\prime}, R^{\prime \prime}\right)$ is the $\left(R^{\prime}, R^{\prime \prime}\right)$-bimodule with generators $\delta(x)(x \in X)$ and relations $\delta(b)=0(b \in H)$, where $\delta(b)$ is the bimodule expression in the symbols $\delta(x)$ and coefficients $x^{\prime}, x^{\prime \prime}$ obtained by formally expanding $\delta(b)$ under the identities for a derivation.

(iii) Given K-algebra homomorphisms $R^{\prime} \rightarrow S^{\prime}, R^{\prime \prime} \rightarrow S^{\prime \prime}$, we bave 


$$
T\left(R ; S^{\prime}, S^{\prime \prime}\right) \simeq S^{\prime} \otimes_{R^{\prime}} T\left(R ; R^{\prime}, R^{\prime \prime}\right) \otimes_{R^{\prime \prime}} S^{\prime \prime}
$$

(iv) Suppose $W$ is a two-sided ideal contained in botb the kernels $U, V$ of $R \rightarrow R^{\prime}$ and $R \rightarrow R^{\prime \prime}$, so that we bave induced bomomorpbisms $R / W \rightarrow R^{\prime}$, $R / W \rightarrow R^{\prime \prime}$. Then $T\left(R / W ; R^{\prime}, R^{\prime \prime}\right) \simeq T\left(R ; R^{\prime}, R^{\prime \prime}\right) / R^{\prime} \delta(W) R^{\prime \prime}$. If W $\subseteq U V$, then $T\left(R / W ; R^{\prime}, R^{\prime \prime}\right) \simeq T\left(R ; R^{\prime}, R^{\prime \prime}\right)$. If the maps $R \rightarrow R^{\prime}, R \rightarrow R^{\prime \prime}$ are surjective, then $R^{\prime} \delta(W) R^{\prime \prime}=\delta(W)$.

Proof. (i) is clear.

(ii) Let $T$ be the $\left(R^{\prime}, R^{\prime \prime}\right)$-bimodule with generators $\delta(x)(x \in X)$ and relations $\delta(b)=0(b \in H)$. Since all these relations hold in $T\left(R ; R^{\prime}, R^{\prime \prime}\right)$ we get a natural homomorphism $\nu: T \rightarrow T\left(R ; R^{\prime}, R^{\prime \prime}\right)$ which is clearly onto. Also, as is easily seen from Lemma 2 , the map $x \rightarrow \delta(x)$ extends to a derivation $R \rightarrow T$. So, by the universality of $T\left(R ; R^{\prime}, R^{\prime \prime}\right), \nu$ must be an isomorphism.

(iii) This follows from (i), since $T\left(R ; S^{\prime}, S^{\prime \prime}\right)$ and $S^{\prime} \otimes_{R^{\prime}} T\left(R ; R^{\prime}, R^{\prime \prime}\right) \otimes_{R^{\prime \prime}} S^{\prime \prime}$ are presented on the same generators and relations.

(iv) The first claim is immediate from the presentation given in (ii), and the second claim follows since $\delta(U V)=0$. For the last claim, note that any element $a^{\prime} \delta(w) b^{\prime \prime}(a, b \in R, w \in W)$ can be written as $\delta(a w b)$, since the fact that $w \in U$ and $w \in V$ causes the first and last terms of the expansion of this element to vanish, leaving the desired term.

From (i) of the preceding lemma we have:

Corollary 4. If $R$ is the free associative $K$-algebra $K\langle X\rangle$, then for any $R^{\prime}, R^{\prime \prime}, T\left(R ; R^{\prime}, R^{\prime \prime}\right)$ is the free $\left(R^{\prime}, R^{\prime \prime}\right)$-bimodule on $\{\delta(x) ; x \in X\}$.

Corollary 5. Let $R, R^{\prime}, R^{\prime \prime}$ be given and let $I$ be a set of elements of $R$ such that for all $z \in I, z^{\prime}$ and $z^{\prime \prime}$ are invertible in $R^{\prime}$ and $R^{\prime \prime}$. Let $R\left\langle I^{-1}\right\rangle$ denote the universal inverting ring for $I$ (the ring obtained from $R$ by formally adjoining inverses to elements of $I)$. Then $T\left(R\left(I^{-1}\right) ; R^{\prime}, R^{\prime \prime}\right) \simeq T\left(R ; R^{\prime}, R^{\prime \prime}\right)$. In particular, if $R$ is any mixed free algebra on the set $X$, then $T\left(R ; R^{\prime}, R^{\prime \prime}\right)$ is the free $\left(R^{\prime}, R^{\prime \prime}\right)$ bimodule on $\{\delta(x), x \in X\}$.

Proof. By definition, we have maps $R\left\langle I^{-1}\right\rangle \rightarrow R^{\prime}, R\left\langle I^{-1}\right\rangle \rightarrow R^{\prime \prime}$ which extend $R \rightarrow R^{\prime}, R \rightarrow R^{\prime \prime}$. By Lemma 3, part (ii), we obtain $T\left(R\left\langle I^{-1}\right\rangle ; R^{\prime}, R^{\prime \prime}\right)$ from $T\left(R ; R^{\prime}, R^{\prime \prime}\right)$ by adding for each $z^{-1} \in I^{-1}$ a new generator $\delta\left(z^{-1}\right)$ and a relation $\delta\left(z^{-1}\right)=-z^{1-1} \delta(z) z^{\prime \prime-1}$. This relation allows us to eliminate the generator $\delta\left(z^{-1}\right)$. Alternatively, we may observe that the images of elements $z \in I$ under the map of $R$ into

$$
\left(\begin{array}{ll}
R^{\prime \prime} & 0 \\
T\left(R ; R^{\prime}, R^{\prime \prime}\right) & R^{\prime}
\end{array}\right)
$$


are invertible, so this map extends uniquely to a map from $R\left\langle I^{-1}\right\rangle$. Thus $T\left(R ; R^{\prime}, R^{\prime \prime}\right)$, with the induced derivation of $R\left\langle I^{-1}\right\rangle$, has the universal property.

Suppose $R$ is a $K$-algebra and $T$ an $(R, R)$-bimodule, $\delta: R \rightarrow T$ a derivation, and $V$ a 2-sided ideal of $R$. Then a derivation $\bar{\delta}: R \rightarrow T / T V$ is induced. One finds, further, that the restriction $\left.\delta\right|_{V}$ will be a left $R-$ module homomorphism, which a derivation generally is not:

$$
\bar{\delta}(r v)=\bar{\delta}(v)+\bar{\delta}(r) v=r \delta(v)+T V=r \bar{\delta}(v) \quad(r \in R, v \in V) .
$$

In the statement that $\delta$ is a derivation, we can think of $T / T V$ either as an $(R, R)$ or an $(R, R / V)$-bimodule. More generally, if $V$ is a left ideal of $R$, we can consider $T / T V$ as a left $R$-module; then it will be meaningless to ask whether $\bar{\delta}$ is a derivation, but $\left.\bar{\delta}\right|_{V}$ will still be a left module homomorphism.

If $U$ is an ideal (or more generally, a right ideal) of a ring $R$, a submodule $B$ of a left $R$-module $A$ is called $U$-pure if $B \cap U A=U B$. Clearly, any direct summand in $A$ is $U$-pure for all $U$.

From the above remarks we have:

Lemma 6. Suppose $R$ is a K-algebra, $T$ an $(R, R)$-bimodule, $\delta: R \rightarrow T$ a derivation, and $V, U$ ideals of $R$ (more generally: $U$ a right ideal and $V$ a left ideal). Suppose:

(i) the map $\left.\bar{\delta}\right|_{V}: V \rightarrow T / T V$ is $1-1$, and

(ii) the submodule $\bar{\delta}(V) \subseteq T / T V$ is U-pure.

Then the kernel of the map $\bar{\delta}: V \rightarrow T /(U T+T V)$ is $U V$, and bence the kernel of the map

$$
R \rightarrow\left(\begin{array}{lc}
R / V & 0 \\
T / U T+T V & R / U
\end{array}\right)
$$

(wbich is a ring bomomorpbism of $U$ and $V$ are 2-sided ideals) is also UV.

We now come to our one nontrivial computational result.

Lemma 7. Let $R$ be the free K-a lgebra on a set $X, V$ a proper left ideal of $R$, and let $T, \delta$ denote $T(R ; R, R)$ and the associated derivation $R \rightarrow T$. Then $\left.\bar{\delta}\right|_{V}: V \rightarrow T / T V$ is $1-1$, and the image of this map is a direct summand in $T / T V$ as a left R-module.

Proof. A $K$-basis for $R$ is given by the set $B$ of all monomials (words) in the elements of $X$. Also, by Corollary $4, T$ has for $K$-basis the set $B \delta(X) B$ of all products $b_{1} \delta(x) b_{2},\left(b_{1}, b_{2} \in B, x \in X\right)$, and hence for left $R$-basis the set $\delta(X) B$ of all products $\delta(x) b$. Let us choose any well-ordering of $X$. This induces a well-ordering on $\tilde{B}$, by taking words of greater length to be greater than words of smaller length, and using lexicographic order for words of equal length. Likewise, we get a well-ordering on $\delta(X) B$. These in turn give us a well-ordering 
of $B \delta(X) B$, where we first count the $\delta(X) B$-part, then the $B$-part on the left.

Given any element $z \in R-\{0\}$ (resp. $T-\{0\}$ ) we define its leading monomial to be the greatest element of $B$ (resp. $B \delta(X) B$ ) occurring with nonzero scalar coefficient in $z$.

Let $C$ denote the set of elements $c \in B$ such that (1) $C$ is the leading monomial of an element of $V-\{0\}$, but (2) $c$ is not a proper left multiple in $B$ of any other monomial satisfying (1). Thus, the leading monomial of any element of $V-\{0\}$ can be written uniquely as $b c(b \in B, c \in C)$. It is easily deduced that if we choose for each $c \in C$ an element $a_{c} \in V$ with leading monomial $c$, then $\left\{a_{c}\right\}$ gives a free basis for $U$ as a left $R$-module. It is also easy to verify that $T V$ will be left $R$-free with basis $\left\{\delta(x) b a{ }_{c} \mid x \in X, b \in B, c \in C\right\}$, and that these basis elements have distinct leading terms $\delta(x) b c$. Hence $T / T V$ will be left $R$-free and have for $R$-basis the images of the elements of $\delta(X) B-\delta(X) B C$; and hence for $K$-basis, the images of the elements of $B \delta(X) B-B \delta(X) B C$.

We now show that the elements $\delta\left(b a_{c}\right)$ have distinct leading terms modulo $T V$, and hence are $K$-independent modulo $T V$. Since $1 \notin V$ each element $c \in C$ can be written uniquely $c=x_{c} d_{c}\left(x_{c} \in X, d_{c} \in B\right)$. It is easily deduced that the leading term of $\delta\left(a_{c}\right)$ is $\delta\left(x_{c}\right) d_{c}$; and because no element of $C$ is a proper left multiple of any other, this monomial is not in $\delta(X) B C$. More generally, given $b \in B$, we can write $\delta\left(b a_{c}\right)=\delta(b) a_{c}+b \delta\left(a_{c}\right)$. The first of these terms lies in $T V$, so modulo $T V, \delta\left(b a_{c}\right)$ is equal to an element with leading term $b \delta\left(x_{c}\right) d_{c}$. This is not the leading term of any element of $T V$, so the element can be "reduced" no further.

We conclude that the elements $\bar{\delta}\left(a_{c}\right)$ are left $R$-linearly independent in $T / T V$, giving the desired injectivity, and that together with the images of the monomials of $\delta(X) B$ that are not of the form $\delta(x) b c$ or $\delta\left(x_{c}\right) d_{c}$, they will give a left $R$-basis of $T / T V$; hence $\bar{\delta}(V)$ is a direct summand in this module.

We can now prove the simplest version of our main theorem.

Theorem 1. Let $R$ be a free associative algebra, and $U, V, 2$-sided ideals of $R$. Then the kernel of the bomomorpbism

$$
R \rightarrow\left(\begin{array}{lc}
R / V & 0 \\
T(R ; R / U, R / V) & R / U
\end{array}\right)
$$

is UV.

Proof. If $V=R$ the result is obvious. If $V$ is a proper ideal, then writing $T(R ; R, R)=T$, we deduce from Lemma 3, part (iii) that $T(R ; R / U, R / V) \simeq$ $T /(U T+T V)$. By Lemmas 6 and 7 , the kernel of the map $\left.\overline{\bar{\delta}}\right|_{V}$ of $V$ into this bimodule is $U V$. Hence the kernel of the ring homomorphism in question, $U \cap V \cap$ Ker $\overline{\bar{\delta}}$, is also UV. 
More generally:

Theorem 2. Let $R$ be any K-algebra, and $U, V$ ideals of $R$. Then the kernel of the map

is UV.

$$
R \rightarrow\left(\begin{array}{lc}
R / V & 0 \\
T(R ; R / U, R / V) & R / U
\end{array}\right)
$$

Proof. Write $R$ as a homomorphic image of a free $K$-algebra $F$, and let $W, \tilde{U}$, $\tilde{V}$ denote the kernels of the maps of $F$ into $R, R / U, R / V$. Then Lemma 3(iv) tells us that we can write $T(R ; R / U, R / V)=T(F / W ; F / \tilde{U}, F / \tilde{V})$ as $T(F ; F / \tilde{U}, F / \tilde{V}) / \tilde{\delta}(W)$. Hence the kernel of the map $\tilde{\delta}^{\prime}: F \rightarrow T(R ; R / U, R / V)$ is $\operatorname{Ker} \tilde{\delta}+W$. But $\operatorname{Ker} \tilde{\delta} \cap \tilde{U} \cap \tilde{V}=\tilde{U} \tilde{V}$ by the preceding theorem, so $\tilde{U} \cap \tilde{V} \cap \operatorname{Ker} \tilde{\delta}^{\prime}$ $=\tilde{U} \tilde{V}+W$; so the kernel of the induced map $\delta: R \rightarrow T(R ; R / U, R / V)$, intersected with $U$ and $V$, is exactly $U V$.

In the above theorem, $R$ was an arbitrary $K$-algebra, but the maps of $R$ into the rings that played the roles of $R^{\prime}$ and $R^{\prime \prime}$ had to be assumed surjective. We can remove the surjectivity assumption if we add some restrictions on $R$. Let us call an $R$-bimodule $A$ "biflat" if for any monomorphisms of left and right $R$-modules, $P \rightarrow P^{\prime}$ and $Q \rightarrow Q^{\prime}$, we get a monomorphism $P \otimes_{R} A \otimes_{R} Q \rightarrow P^{\prime} \otimes_{R} A \otimes_{R} Q^{\prime}$. This is equivalent to: For every right $R$-module $P$, the right $R$-module $P \otimes_{R} A$ is flat, and for every left $R$-module $Q$, the left $R$-module $A \otimes_{R} Q$ is flat. In particular, it is not hard to show that any free $R$-bimodule, i.e. a direct sum of copies of $R \otimes_{K} R$, is $R$-biflat. (This, however, uses the fact that $K$ is a field, so that all $K$-modules are $K$-flat. If, instead, $R=K=Z$, the integers, then the free $R$-bimodule $Z \otimes_{Z} Z=Z$ is not biflat: if $Z_{n} \rightarrow Z_{n}$ is the identity map of the integers modulo $n$, and $Z \rightarrow Q$ is the inclusion of the integers in the rationals, then $Z_{n} \otimes Z \otimes Z \rightarrow Z_{n} \otimes Z \otimes Q$ is the zero map $Z_{n} \rightarrow 0$.)

Theorem 3. Let $R$ be any K-algebra sucb that $T=T(R ; R, R)$ is $R$-biflat (e.g., a mixed free K-algebra), and let $R \rightarrow R^{\prime}, R \rightarrow R^{\prime \prime}$ be K-algebra bomomorphisms with kernels $U, V$. Then the kemel of the homomorphism

is UV.

$$
R \rightarrow\left(\begin{array}{ll}
R^{\prime \prime} & 0 \\
T\left(R ; R^{\prime}, R^{\prime \prime}\right) & R^{\prime}
\end{array}\right)
$$

Proof. By the preceding theorem, UV will be the kernel of the map

$$
R \rightarrow\left(\begin{array}{lc}
R / V & 0 \\
T(R ; R / U, R / V & R / U
\end{array}\right)
$$

By Lemma 3(iii), $T(R ; R / U, R / V)$ and $T\left(R ; R^{\prime}, R^{\prime \prime}\right)$ can be written as $(R / U) \otimes_{R}$ $T \otimes_{R}(R / V)$ and $R^{\prime} \otimes_{R} T \otimes_{R} R^{\prime \prime}$, respectively. By biflatness of $T$, the former object will embed in the latter, hence 


$$
\left(\begin{array}{ll}
R^{\prime} V & 0 \\
T(R ; R / U, R / V) & R / U
\end{array}\right) \text { embeds in }\left(\begin{array}{ll}
R^{\prime \prime} & 0 \\
T\left(R ; R^{\prime}, R^{\prime \prime}\right) & R^{\prime}
\end{array}\right)
$$

so the kernel of the map of $R$ into the latter ring is also $U V$.

However, the common conclusion of Theorems 2 and 3 is not true for arbitrary $R$ and arbitrary $R^{\prime}, R^{\prime \prime}$. Let $R=K[x, y] /(x y)$, and $R^{\prime}=R^{\prime \prime}=K\left[x, x^{-1}\right]$ with $x \rightarrow x$ and $y \rightarrow 0$. Then $U=V=(y)$, and $y \notin U V$. However, in $T\left(R ; R^{\prime}, R^{\prime \prime}\right)$, $0=\delta(x y)=x(\delta y)+(\delta x) y=x(\delta y)+(\delta x) 0=x(\delta y)$, hence since $x$ is invertible in $R^{\prime}, 0=\delta y$. Hence

$$
y \in \operatorname{Ker}\left(R \rightarrow\left(\begin{array}{ll}
R^{\prime} & 0 \\
T & R^{\prime}
\end{array}\right)\right)
$$

A number of variants to Theorem 3 can be proved in the same way. E.g., we might assume that $T$ is flat only on one side, and that one of the given maps $R \rightarrow R^{\prime}, R^{\prime \prime}$ is surjective; or we might assume $R$ arbitrary but impose conditions on the right and left $R$-module structures of $R^{\prime}$ and $R^{\prime \prime}$ (see [3, Corollary 4]). In any case, as a corollary to Theorem 2 , we have

Theorem 4. Let $R$ be any K-algebra, and $U, V, N$ ideals of $R$ sucb that $U V \subseteq N \subseteq U \cap V$. Then $N$ is the kernel of the map

$$
R \rightarrow\left(\begin{array}{lc}
R / V & 0 \\
T(R / N ; R / U, R / V) & R / U
\end{array}\right)
$$

The above arguments are remarkable in that they give nontrivial results about arbitrary $K$-algebras using structure-theoretic considerations about free $K$-algebras. The one parallel which comes to mind is the Noetherian property of finitely generated commutative algebras, proved by first looking at free commutative algebras.

2. Going back to our motivating example, let $G$ be a free group on free generators $\{x \mid x \in X\}$, and let $R$ be a normal subgroup of $G$. Let $x, g$ be the augmentation ideals of $R$ and $G$ in the group algebra $K G$ over a field of characteristic zero. Since $K G / g=K, T(K G ; K G / g, K G / \mathfrak{x})$ is just a free $K G / \mathfrak{x}$ module, i.e. a free $K(G / R)$ module with basis $\{\delta(x)\}(x \in X)$. Since both $\delta$ and the Fox derivative taken modulo $R$ have the universal property, they coincide. (This can also be seen combinatorially.) This shows that the Magnus representation for $G /[R, R]$ is embedded in the representation for $K G / g x$ given by Theorem 1 . Alternatively, using the fact that $[8, \mathrm{p} .53] \mathrm{Ker}(G \rightarrow K G / g x)=[R, R]$, we could derive the Magnus representation from Theorem 1. We use this to represent some other groups in the next section.

Theorem 2 can be stated solely in terms of the universal derivation $\delta$. (See Lyndon [15] for the corresponding theorem about free groups.) 
Theorem 5. Let $R$ be a K-algebra with two ideals $U, V$ and let $R^{\prime}=F / U$, $R^{\prime \prime}=F / V$. Let $w \in U \cap V$. Then $w \in U V$ if and only if $\delta(w)=0$ in $T\left(R ; R^{\prime}, R^{\prime \prime}\right)$.

Let $R, U, V, R^{\prime}, R^{\prime \prime}$ be as in Theorem 5. We can extend the representation given by Theorem 2 to some other ideals. We turn the abelian group $T\left(R ; R^{\prime}, R^{\prime \prime}\right)$ $\oplus T\left(R ; R^{\prime \prime}, R^{\prime}\right)$ into a ring with zero multiplication. It is then clear that we have a homomorphism

$$
\nu: R \rightarrow\left(\begin{array}{lc}
R^{\prime \prime} & T\left(R ; R^{\prime \prime}, R^{\prime}\right) \\
T\left(R ; R^{\prime}, R^{\prime \prime}\right) & R^{\prime}
\end{array}\right)
$$

If $z$ is in the kernel of $\nu$ then $z \in U \cap V$ and its derivatives in both $T\left(R ; R^{\prime}, R^{\prime \prime}\right)$ and $T\left(R ; R^{\prime \prime}, R^{\prime}\right)$ are zero. Thus by Theorem 5, z $\in U V \cap V U$. So $U V \cap V U=\operatorname{Ker} \nu$.

Still keeping the same notation, let $\bar{R}=R / U V$, and denote the natural map $R \rightarrow \bar{R}$ by $r \rightarrow \bar{r}$. By Lemma 3(iv), $T\left(R ; R^{\prime}, R^{\prime \prime}\right)=T\left(\bar{R} ; R^{\prime}, R^{\prime \prime}\right)$ and we have an induced derivation (which is universal) $\bar{\delta}: \bar{R} \rightarrow T\left(\bar{R} ; R^{\prime}, R^{\prime \prime}\right)$. If $\bar{z} \in \overline{U \cap V,}$ then $z^{\prime}=z^{\prime \prime}=0$. Hence

$$
\bar{\delta}\left(\overline{r_{1} z r_{2}}\right)=r_{1}^{\prime} \bar{\delta}(\bar{z}) r_{2}^{\prime \prime}, \quad r_{1}, r_{2} \in R, z \in U \cap V .
$$

Now, left and right multiplication by elements of $\bar{R}$ induces an $\left(R^{\prime}, R^{\prime \prime}\right)$-bimodule structure on $\overline{U \cap V}$, and the above equation states that $\bar{\delta}$ restricted to $\overline{U \cap V}$ is a bimodule homomorphism. Since $\operatorname{Ker} \bar{\delta}=0$ we have from Theorem 5:

Theorem 6. Let $R$ be a K-algebra, $U, V$ two ideals of $R$ and $R^{\prime}=F / U$, $R^{\prime \prime}=F / V$. Then $U \cap V / U V$ is isomorpbic (as $\left(R^{\prime}, R^{\prime \prime}\right)$-bimodule) to a submodule of $T\left(R ; R^{\prime}, R^{\prime \prime}\right)$. The embedding is given by $z+U V \rightarrow \delta(z)$.

In particular, if $R$ is a mixed free algebra, $T\left(R ; R^{\prime}, R^{\prime \prime}\right)$ is a free $\left(R^{\prime}, R^{\prime \prime}\right)$. bimodule. So we have

Theorem 7. Let $K\langle X\rangle$ be a mixed free algebra, and let $U, V$ be two ideals of $K\langle X\rangle$. Then $U \cap V / U V$ can be embedded in a free $(F / U, F / V)$-bimodule.

As an easy application we prove

Theorem 8 (Simple identity theorem for Lie elements, cf. Lyndon [15]). Let $F=K\langle X\rangle$ be a free algebra and let $u \neq 0$ be a Lie element of $F$. Let $U$ be the ideal of $F$ generated by $u$. Then $U / U^{2}$ is the free $F / U$-bimodule generated by $u+U^{2}$.

Proof. If $L$ is the Lie algebra on the generators of $F$ with the single relation $u=0$, then $F / U$ is the universal enveloping algebra of $L$. Thus $(F / U)^{\text {opp }} \otimes_{K} F / U$ is the universal enveloping algebra of $L^{\text {opp }} \oplus L$. Thus 
$(F / U)^{\text {OPP }} \otimes_{K} F / U$ is an integral domain [10, p. 166], so a one-generator nonzero submodule of a free $F / U$ bimodule is again free. Since $\cap U^{i}=0$ (this is true for any proper ideal of $F(\operatorname{Cohn}[6]))$ and since $U / U^{2}$ is generated by $u+U^{2}$, $u+U^{2} \neq 0$. By Theorem $7, U / U^{2}$ can be embedded in a free $F / U$-bimodule. It follows that $U / U^{2}$ is free.

(This theorem has been proved by Warren Dicks. See also J. Labute [11].)

If $\mu$ is the $U$-adic filtration on $F$ (the filtration defined by the powers of $U$ ) then the associated graded algebra $g r F$ is the tensor algebra on the $F / U$-bimodule $U / U^{2}$. (This is true for any ideal of $F$ (Cohn [6]).) So in this case, gr $F$ is the tensor algebra in one generator over the enveloping algebra of $L$. In particular $U^{n} / U^{n+1}$ is also a free $F / U$-bimodule, since

$$
U^{n} / U^{n+1} \simeq U / U^{2} \otimes_{F / U} U / U^{2} \otimes_{F / U} \cdots \otimes_{F / U} U / U^{2} .
$$

IV. Embeddings in matrices. Let $R$ be a $K$-algebra. To simplify terminology, let us say that $R$ is embeddable in matrices if there exist a commutative $K$-algebra $A$, and, for some integer $n$, an injective $K$-homomorphism of $R$ into $A_{n}$, the algebra of $n \times n$ matrices over $A$. In an important special case, the formal matrices of our representation give rise to embeddings in matrices.

Theorem 9. Let $F=K\langle X\rangle$ be a mixed free algebra and let $U$ and $V$ be ideals of $F$. If $F / U$ and $F / V$ are embeddable in matrices, so is $F / U V$. Specifically, . if $F / U$ and $F / V$ are embeddable in $k \times k$ and $l \times l$ matrices respectively, then $F / U V$ is embeddable in $k+l \times k+l$ matrices.

Proof. This proof is Bergman's improved version.

For any ring $R$, we write $R_{i j}$ for the additive group of $i \times j$ matrices over $R_{\text {. }}$ Let $A, B$ be commutative $K$-algebras and let $C=A \otimes_{K} B$. Then, qua $\left(A_{k k}, B_{l l}\right)-$

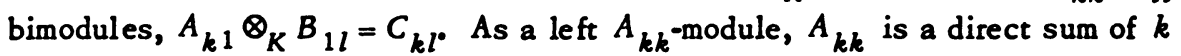
copies of $A_{k 1}$ and as a right $B_{l l^{-m o d u l e}, B_{l l}}$ is a direct sum of $l$ copies of $B_{1 l^{\circ}}$ So, as an $\left(A_{k k^{\prime}} B_{l l}\right)$-bimodule, $A_{k k} \otimes_{K} B_{l l}$ is isomorphic to a direct sum of $k l$ copies of $C_{k l^{\circ}}$ (To avoid confusion, let us note that the left and right actions by $A_{k k}$ and $B_{l l}$ are given by left and right multiplications by the images $A_{k k} \otimes 1$ and $1 \otimes B_{l l}$ of $A_{k k}$ and $B_{l l}$ respectively.)

Now, as an $\left(A_{k k}, B_{l l}\right)$-bimodule, $C_{k l}$ is generated by any of the matrices $e_{i j}$ with a 1 in the $(i, j)$ position and zeros elsewhere. For each $x \in X$, let us adjoin to $C k l$ indeterminates $t_{x}^{i j}, 1 \leq i \leq k ; 1 \leq j \leq l$, and let $C^{\prime}$ be the polynomial ring $C\left[t_{x}^{i j}\right]$. Then for each $x \in X$, the generic matrix $T_{x}=\left(t_{x}^{i j}\right)$ generates a free one-generator $\left(A_{k k^{\prime}} B_{l l}\right)$-bimodule, since $A_{k k} T_{x} B_{l l}$ is the direct sum

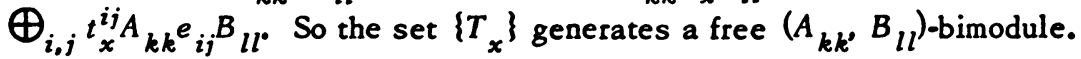

Now, by the assumptions of the theorem, there are homomorphisms $\pi_{1}: F \rightarrow$ $A_{k k}, \pi_{2}: F \rightarrow B_{l l}$ with kernels $U, V$ respectively. Since $T\left(F ; A_{k k}, B_{l l}\right)$ is 
isomorphic, as $\left(A_{k k^{\prime}} B_{l l}\right)$-bimodule, to the bimodule generated by the matrices $T_{\boldsymbol{x}}$, it follows from Theorem 3 that the map defined by

$$
x \rightarrow\left(\begin{array}{cc}
1 \otimes x \pi_{2} & 0 \\
T_{x} & x \pi_{1} \otimes 1
\end{array}\right),
$$

from $F$ to $C_{k+l, k+l}^{\prime}$ has kernel $U V$. This proves the theorem.

We now apply Theorem 9 to algebras satisfying a polynomial identity (PI algebras). For convenience we assume that the field $K$ is infinite. (Otherwise we would have to replace $K$ by some infinite extension, its algebraic closure, say.)

Let $M$ be a square matrix whose nonzero entries are algebraically independent over $K$, and let $M_{x}(x \in X)$ be replicas of $M$ such that the nonzero entries of $\left\{M_{x}\right\}$ are all algebraically independent. Let $R$ be the algebra generated by $\left\{M_{x}\right\}$. If every element of $R$ has a zero entry whenever $M$ has a zero entry, we will say that $M$ is a generic matrix and that $R$ is an algebra of generic matrices. A generic matrix without any zero entries will be called a full generic matrix.

$A$ variety of algebras is the class of all algebras satisfying a given set of polynomial identities. It is well known [1] that the algebras of full generic $n \times n$ matrices are the free algebras in the variety generated by the ring $K_{n}$ of $n \times n$ matrices over $K$. Further, these are the only semisimple free PI algebras. Likewise if $M$ is generic, then $R=K\left\langle u_{x}\right\rangle$ is clearly a free algebra in the variety generated by the matrices over $K$ of the same size as $M$ and with a zero entry whenever $M$ has a zero entry. Thus to each algebra of generic matrices on generators $M_{x}$, there corresponds a $T$-ideal $M$ of the free algebra $K\langle X\rangle$.

If $K\langle X\rangle / \mathfrak{m}_{1}$ and $K\langle X\rangle / \mathrm{m}_{2}$ are algebras of generic matrices, then the proof of Theorem 9 makes it clear that $K\langle X\rangle / \mathfrak{m}_{1} \mathfrak{m}_{2}$ is al so an algebra of generic matrices: If $K\langle X\rangle / \mathfrak{m}_{1}$ and $K\langle X\rangle / \mathfrak{m}_{2}$ are represented as matrices over polynomial rings $K\left[S_{1}\right]$ and $K\left[S_{2}\right]$ with $S_{1} \cap S_{2}=\varnothing$, then we may dispense with the tensor signs in the representation of $K\langle X\rangle / \mathfrak{m}_{1} \mathfrak{m}_{2}$. And, by construction, the lower left-hand corner in the image of $x \in X$ is filled with new indeterminates. So the image of $x$ is again generic. In particular, if $t_{n}$ is the $T$-ideal of $K\langle X\rangle$ corresponding to an algebra of full $n \times n$ generic matrices, then for any $k, K\langle X\rangle / t_{n}^{k}$ is again an algebra of generic matrices and, a fortiori, is embeddable in matrices. Now, if $R$ is a PI $K$-algebra with nilpotent (Jacobson) radical, then $R$ is a homomorphic image of some $K\langle X\rangle / t_{n}^{k}$.

More generally, if $N$ is the upper nil radical of the PI algebra $R$, then $R / N$ has no nil ideals, so the polynomial ring $(R / N)[y]$ is a semisimple PI algebra and hence is embeddable in matrices. Thus if $N^{k}=0, R$ is also a homomorphic image of some $K\langle X\rangle / t_{n}^{k}$. Thus we have shown

Theorem 10. If $R$ is a $P I K$-algebra with nilpotent upper nil radical and $K$ is infinite, then, for some $k, R$ satisfies all the identities of $k \times k$ matrices over $K$. 
Note that by an observation of Latyshev [12] a finitely generated PI algebra $\boldsymbol{R}$ whose radical is a finitely generated $R$-bimodule has a nilpotent radical. So, for finitely generated PI algebras, $R / J(R)$ commutative implies that $R$ satisfies all the identities of $k \times k$ matrices and in particular that $R$ satisfies some standard identity. These remarks point out the close relationship among the following three open problems: Does every finitely generated PI algebra have a nilpotent radical? Does every finitely generated PI algebra satisfy a standard identity? If $X$ is finite, is $t_{n}$ a finitely generated $K\langle X\rangle$ bimodule? At present we can only say that if the second question has a negative answer, so do the other two.

Several special cases of Theorem 9 are of interest. If we start with $F^{\circ}$, a free algebra without one, set $U=V=F^{\circ}$ and iterate the procedure of Theorem 9, we obtain strictly lower triangular (zero on the diagonal) matrices. Thus we have

Theorem 11. The algebra of strictly lower triangular generic $k \times k$ matrices on $d$ generators is the free nilpotent algebra of class $k-1$ and rank $d$.

Likewise, with $F$ a free algebra and $U=V=t_{1}=[F, F]$, the commutator ideal, we find

Theorem 12. The algebra of lower triangular generic $k \times k$ matrices on $d$ generators is the free algebra of rank $d$ in the variety of algebras whose commutator ideal is nilpotent of class $k-1$.

If $F$ is now the group algebra $K G$ of the free group $G$, and $C$ is the augmentation ideal of the commutator subgroup $[G, G]$ of $G$, then we find that lower triangular matrices, where now the diagonal entries are invertible, represent $K G / c^{k}$ faithfully. Since $[8$, p. 53], with char $K=0$ and $g$ the augmentation ideal of $G, \operatorname{Ker}\left(G \rightarrow K G / c^{k}\right)=\operatorname{Ker}\left(G \rightarrow K G / g c^{k-1}\right)=\gamma_{k}\left(G^{\prime}\right)$, the $k$ th term of the lower central series of $[G, G]$, we have

Theorem 13. If char $K=0$, then generic lower triangular $k \times k$ matrices with invertible entries on the diagonal freely generate a free group in the variety of groups whose commutator is nilpotent of class $k-1$. The $(1,1)$-entry in each generator may also be taken to be 1.

This theorem has also been proved by N. Gupta and K. Gupta [9]. Note that since $\operatorname{Ker}\left(G \rightarrow K G / g^{k}\right)=\gamma_{k}(G)$ we have also shown that lower triangular generic matrices with 1 on the diagonal freely generate free nilpotent groups. This is well known.

Coming back to a free algebra $F$, it is not true that we obtain all algebras of generic matrices by using the construction of Theorem 9 starting with the $T$-ideals $t_{n}(n=1,2, \ldots)$ and allowing multiplication and intersection as operations. Indeed the generic matrix 


$$
\left(\begin{array}{llll}
x_{1} & 0 & 0 & 0 \\
z_{1} & y_{1} & 0 & 0 \\
0 & 0 & x_{2} & 0 \\
t & 0 & z_{2} & y_{2}
\end{array}\right)
$$

cannot be obtained in this way, even if we allow similarity transformations. This matrix however still corresponds to the $T$-ideal $t_{1}^{2}$ and we have not been able to construct an algebra of generic matrices which does not correspond to a $T$-ideal in the above family.

UP to now, we have only shown how to embed free PI algebras in matrices. We now exhibit a variety in which every algebra is embeddable in matrices.

Theorem 14. Let $R$ be a K-algebra with $[R, R]^{2}=0$. Then $R$ is embeddable in $2 \times 2$ lower triangular matrices.

Proof. Present $R$ via $0 \rightarrow N \rightarrow K\langle X\rangle \rightarrow R \rightarrow 0$, and let $U$ be the inverse image of $[R, R]$. Then, by assumption, $U^{2} \leq N \leq U$. Thus if $\left.A=K\langle X\rangle / U \simeq R / R, R\right]$, by Theorem $4, N$ is the kernel of the map

$$
K\langle x\rangle \rightarrow\left(\begin{array}{ll}
A & 0 \\
T(F / N ; A, A) & A
\end{array}\right) .
$$

Consider $T=T(F / N ; A, A)$ as a right $A \otimes A$ module. Te can find a commutative $K$-algebra $C$ which contains both $A \otimes A$ and $T$. We may construct $C$ by defining on the direct sum $(A \otimes A) \oplus T$ the multiplication $\left(b_{1}+t_{1}\right)\left(b_{2}+t_{2}\right)=b_{1} b_{2}+$ $t_{1} b_{2}+t_{2} b_{1} \cdot\left(b_{1}, b_{2} \in A \otimes A ; t_{1}, t_{2} \in T\right.$.) Now, with the obvious identification, $\left(\begin{array}{ll}A & 0 \\ T & A\end{array}\right) \subseteq\left(\begin{array}{ll}C & 0 \\ C & C\end{array}\right)$ which proves the theorem.

This theorem is most likely the best that one can do. If every algebra in the variety $\mathcal{O}$ is embeddable, then $\mathcal{O}$ has degree of commutativity 1 (Amitsur [2]). Also, Small [17] has constructed an algebra $R$ with $J(R)^{3}=0$ and $R / J(R)$ not embeddable in matrices. Using the technique of Lewin [13] one can also find a PI algebra without 1 , such that $R / J(R)$ is commutative, $J(R)^{2} R=0$ and yet $R$ is not embeddable in matrices.

We also obtain some information in the finite dimensional case.

Theorem 15. Let $R$ be a finite dimensional K-algebra sucb that $J(R)^{2}=0$ and $R / J(R)$ is separable and embeddable in $n \times n$ matrices. Then $R$ can be embedded in $2 n \times 2 n$ matrices.

Proof. As above, present $R$ via $0 \rightarrow N \rightarrow K\langle X\rangle \rightarrow R \rightarrow 0$ and let $U$ be the inverse image of $J(R)$. Then by Theorem $1, K\langle X\rangle / U^{2}$ is embedded in

$$
\left(\begin{array}{cc}
K\langle X\rangle / U & 0 \\
T & K\langle X\rangle / U
\end{array}\right)
$$


with $T=T(K\langle X\rangle ; K\langle X\rangle / U, K\langle X\rangle / U)$. By separability $R / J(R)^{\text {opp }} \otimes_{K} R / J(R)$ is again semisimple so that $T$ is completely reducible. In particular $N / U^{2}$ is a direct summand of $T$. Let $\rho: T \rightarrow N / U^{2}$ be the projection. Then it is easily verified that $R$ is isomorphic to the algebra generated by the matrices

$$
\left(\begin{array}{lc}
x+U & 0 \\
\delta(x)-\delta(x) \rho & x+U
\end{array}\right),
$$

and thus $R$ is actually embedded in $K\langle X\rangle / U^{2}$. We then apply Theorem 9 to complete the proof.

Note that in the above situation, it is quite easy to prove Wedderburn's principal theorem: If $T=U / U^{2} \oplus S$, and $\sigma$ is the projection of $T$ onto $S$, then $U / U^{2}$ is complemented by the algebra generated by the matrices

$$
\left\{\left(\begin{array}{cc}
x+U & 0 \\
\delta(x) \sigma & x+U
\end{array}\right), x \in X\right\} .
$$

This algebra is isomorphic to $F / U$. So $F / U^{2}$ splits over its radical $U / U^{2}$. So $F / N$ splits over $U / N$ i.e., $R$ splits over its radical. The full Wedderburn principal theorem follows by induction on the nilpotency class of the radical.

V. Two applications to varieties. Recall that if $F=K\langle X\rangle$ is a free algebra, a $T$-ideal $t$ of $F$ is an ideal which is fully invariant, i.e., $t \eta \subseteq t$ for any endomorphism $\eta$ of $F$. It is not known whether $F$ has ACC on $T$-ideals. For the proof of the next theorem, we refer the reader to D. Cohen's paper [4]. With a slight modification which we indicate below, Cohen's proof of his Theorem $C$ yields

Theorem 16. Let $F=K\langle X\rangle$ be a free algebra. Then $F$ bas $A C C$ on $T$ ideals which contain $[F, F]^{2}$.

Proof. It is clearly sufficient to prove that $B=F /[F, F]^{2}$ has $A C C$ on fully invariant ideals and, as in [4], we may assume that $X$ is countable.

Cohen's proof already shows that $A=B /[B, B]$ has $A C C$ on fully invariant ideals, so it is only necessary to show that $\tilde{B}$ has ACC on fully invariant ideals which are contained in $[B, B]$. By Theorem 2 , such an ideal $t$ is an $(A, A)$-submodule of the free $(A, A)$-bimodule $T=T(F ; A, A)$. Let $A=K\left[X^{\prime}\right]$. Consider $T$ as a right $A^{\text {opp }} \otimes A=A \otimes A$ bimodule. Then $t$ has the property that if

$$
\sum \delta\left(x_{i}\right) p_{i}\left(x_{1}^{\prime} \otimes 1, \ldots, x_{n}^{\prime} \otimes 1 ; 1 \otimes x_{1}^{\prime}, \ldots, 1 \otimes x_{n}^{\prime}\right) \in t,
$$

with $p_{i} \in A \otimes A$, and $\pi$ is a permutation of the natural numbers, then

$$
\sum \delta\left(x_{\pi(i)}\right) p_{i}\left(x_{\pi(1)}^{\prime} \otimes 1, \ldots, x_{\pi(n)}^{\prime} \otimes 1 ; 1 \otimes x_{\pi(1)}^{\prime}, \ldots, 1 \otimes x_{\pi(n)}^{\prime}\right)
$$

is again in $t$. Thus we need to show that $T$ has ACC on such submodules. This is done in the same way as in the proof of Cohen's Proposition 3. However, his 
ordered sets $(V \times J, \leq)$ and $(V \times J, \preceq)$ must here be replaced by $(V \times P, \leq)$ and $(V \times P, \preceq)$ where $P=J \times J$ is the product of two copies of the positive integers with the lexicographic ordering $\left(j_{1}, j_{2}\right)<\left(j_{1}^{\prime}, j_{2}^{\prime}\right)$ if $j_{1}<j_{1}^{\prime}$ or $j_{1}=j_{1}^{\prime}$ and $j_{2}<j_{2}^{\prime}$. This takes care of the fact that even though $A \otimes A$ is the polynomial algebra $K\left[X^{\prime} \otimes 1,1 \otimes X^{\prime}\right]$ (the case covered in Cohen's paper) we are restricted here to endomorphisms of $A \otimes A$ which, if they map $x_{i}^{\prime} \otimes 1$ to $x_{\pi(i)}^{\prime} \otimes 1$, also map $1 \otimes x_{i}^{\prime}$ to $1 \otimes x_{\pi(i)^{\prime}}^{\prime}$ 口

Note that the $T$-ideal $[F, F]^{2}$ is generated, qua $T$-ideal by the single element $u=\left[x_{1}, x_{2}\right] x_{3}\left[x_{4}, x_{5}\right]$. Thus it follows from Theorem 16 that any $T$-ideal which contains the element $u$ is finitely generated, qua $T$-ideal.

Let us now suppose again that $K$ is an infinite field. Let $\mathcal{O}$ be a variety of PI algebras in which every finitely generated algebra is right Noetherian. (These have been classified by Latyshev [12], L'vov [14].) This is equivalent to saying that for every $n$, the $\mathcal{O}$-free $K$-algebra $R$ on $n$ generators is right Noetherian. Since $K$ is infinite, $R$ is homogeneously presented (Amitsur [1]). The following theorem shows that each such $R$, and hence every finitely generated algebra in $\mathcal{C}$, is finitely presented.

Theorem 17. Let $U$ be a bomogeneous ideal of the free algebra $F=$ $K\left\langle x_{1}, \cdots, x_{n}\right\rangle$, and suppose $F / U$ is right Noetberian. Then $U$ is a finitely generated F-bimodule.

Proof. Let $R=F / U$. Since $U \leq f$, the augmentation ideal of $F$, Theorem 1 shows that $U / F U$ is a submodule of a free $(K, R)$-bimodule with basis $\left\{\delta\left(x_{i}\right), i=1, \cdots, n\right\}$. Since $R$ is right Noetherian, $U / f U$ is again a finitely generated $(K, R)$-bimodule. Let $\left\{u_{1}, u_{2}, \ldots\right\}$ be a homogeneous right generating set for $U$. Then for some $n,\left\{u_{1}, \cdots, u_{n}\right\}$ generates $U$ modulo fU. Now assume inductively that for some integer $k$, all the $u_{s}$ having degree $d\left(u_{s}\right)<k$ lie in $\sum_{i=1}^{n} F u_{i} F ;$ and consider any $u_{t}$ with $d\left(u_{t}\right)=k$. We have

so we can write

$$
u_{t} \in U=\sum_{i=1}^{n} u_{i} F+\mathrm{F} U=\sum_{i=1}^{n} u_{i} F+\sum_{s=1}^{\infty} F u_{s} F,
$$

$$
u_{t}=\sum_{i=1}^{n} u_{i} f_{i}+\sum_{s=1}^{\infty}\left(\sum_{j=1}^{m(s)} g_{s j} u_{s} b_{s j}\right),
$$

where we may take each term to be homogeneous of degree $k=d\left(u_{t}\right)$, and where each $g_{s j} \in F$. Now note that for all $s$ such that $d\left(u_{s}\right) \geq k$, the conditions that the terms $g_{s j} u_{s} b_{s j}$ have degree $k$, and that $g_{s j} \in F$, are incompatible, hence all such terms must be zero, and the double summation in (1) must involve only terms $u_{s}$ of degrees $<k$. But by hypothesis, these all lie in $\sum_{i=1}^{n} \bar{F} u_{i} F$, hence by (1), so does $u_{t}$. Thus by induction on $k$, all $u_{s}$ lie in this subideal, so $u_{1}, \cdots, u_{n}$ generate $U$. 
The converse of Theorem 17 is false, even if $F / U$ is a PI algebra, since there are varieties in which the finitely generated free algebras are right, but not necessarily left Noetherian; for example we may choose $U=f[F, F]$.

\section{REFERENCES}

1. S. A. Amitsur, The identities of PI rings, Proc. Amer. Math. Soc. 4 (1953), 27-34. MR 14, 613.

2. - A noncommutative Hilbert basis theorem and subrings of matrices, Trans. Amer. Math. Soc. 149 (1970), 133-142. MR 41 \#3514.

3. G. Bergman and W. Dicks, Universal derivations (to appear).

4. D. E. Cohen, On the laws of a metabelian variety, J. Algebra 5 (1967), 267-273. MR 34 \#5929.

5. P. M. Cohn, On a generalization of the Euclidean algorithm, Proc. Cambridge Philos Soc. 57 (1961), 18-30. MR 22 \#9514; 22, 2547.

6. - On a class of rings with inverse weak algorithm, Math. Z. 117 (1970), 1-6. MR 43 \#4849.

7. R. H. Fox, Free differential calculus. I. Derivation in the free group ring, Ann. of Math. (2) 57 (1953), 547-560. MR 14, 843.

8. K. W. Gruenberg, Cohomological topics in group theory, Lecture notes in Math. 143, Springer-Verlag, New York and Berlin, 1970. MR 43 \#4923.

9. N. Gupta and K. Gupta, On the linearity of free nilpotent by abelian groups, J. Algebra 24 (1973), 293-302.

10. N. Jacobson, Lie algebras, Interscience Tracts in Pure and Appl. Math., no. 10, Interscience, New York, 1962. MR 26 \#1345.

11. J. Labute, Algèbres de Lie et pro-p-groupes définis par une seule relation, Invent. Math. 4(1967), 142-158. MR 36 \#1581.

12. V. N. Latysher, Generalization of the Hilbert theorem on the finiteness of bases, Sibirsk. Mat. Ž. 7 (1966), 1422-1424 = Siberian Math. J. 7 (1966), 1112-1113. MR 34 \#2606.

13. J. Lewin, Some infinitely presented associative algebras and an example of $L$. Small, J. Austral. Math. Soc. (to appear).

14. I. V. $L^{\prime}$ rov, Maximality conditions in algebras with identity relations, Algebra i Logika 8 (1969), 449-459 = Algebra and Logic 8 (1969), 258-263. MR 43 \#4853.

15. R. C. Lyndon, Cohomology of groups with a single defining relation, Ann. of Math. (2) 52 (1950), 650-665. MR 13, 819.

16. W. Magnus, $O_{n}$ a theorem of Marshall Hall, Ann. of Math. 40 (1939), 764-768. MR 1, 44.

17. L. Small, An example in PI rings, J. Algebra 17 (1971), 434-436. MR 42 \#7704. DEPARTMENT OF MATHEMATICS, SYRACUSE UNIVERSITY, SYRACUSE, NEW YORK 13210 